\title{
miR-224-5p regulates the proliferation, migration and invasion of pancreatic mucinous cystadenocarcinoma by targeting PTEN
}

\author{
XIAOBO PENG ${ }^{1 *}$, CHENGTAO GUO $^{1 *}$, YANJUN WU $^{2 *}$, MINGZHEN YING $^{1}$, \\ RENXU CHANG $^{2}$, LELE SONG ${ }^{2}$, LIXING ZHAN ${ }^{2}$ and XIANBAO ZHAN ${ }^{1}$ \\ ${ }^{1}$ Department of Oncology, Changhai Hospital Affiliated to Naval Military Medical University, Shanghai 200433; \\ ${ }^{2}$ CAS Key Laboratory of Nutrition, Metabolism and Food Safety, Shanghai Institute of Nutrition \\ and Health, Shanghai Institutes for Biological Sciences, Chinese Academy of Sciences, \\ University of Chinese Academy of Sciences, Shanghai 200031, P.R. China
}

Received September 30, 2020; Accepted February 18, 2021

DOI: $10.3892 / \mathrm{mmr} .2021 .11985$

\begin{abstract}
Pancreatic mucinous cystadenocarcinoma (MCC) is a rare malignant tumor, with a limited number of studies. The present study aimed to investigate the function and mechanism of microRNA (miR)-224-5p on proliferation, migration and invasion of MCC of the pancreas. Reverse transcription-quantitative PCR was used to explorethe expression of miR-224-5p and the PTEN gene. MTT, wound healing, Transwell and tumorigenesis assays were conducted to investigate the proliferation, migration and invasion of $\mathrm{MCC} 1$ cells in vitro and in vivo. Western blot analysis was employed to test the protein expression of PTEN. The target gene of miR-224-5p was assessed and verified by luciferase assay. miR-224-5p expression was notably higher, while PTEN expression was lower, in $\mathrm{MCCl}$ cells compared with normal tissues and cells. Overexpression of miR-224-5p promoted the proliferation, migration and invasion of MCC and knockdown of miR-224-5p inhibited these functions. Bioinformatics analysis and luciferase assay indicated that PTEN was the direct target gene of miR-224-5p. The negative correlation between miR-224-5p and PTEN was confirmed both in vitro and
\end{abstract}

Correspondence to: Professor Xianbao Zhan, Department of Oncology, Changhai Hospital Affiliated to Naval Military Medical University, 168 Changhai Road, Yangpu, Shanghai 200433, P.R China E-mail: zhanxianbaoch@163.com

Professor Lixing Zhan, CAS Key Laboratory of Nutrition, Metabolism and Food Safety, Shanghai Institute of Nutrition and Health, Shanghai Institutes for Biological Sciences, Chinese Academy of Sciences, University of Chinese Academy of Sciences, 320 Yueyang Road, Xuhui, Shanghai 200031, P.R. China

E-mail: 1xzhansibs@163.com

*Contributed equally

Key words: pancreatic mucinous cystadenocarcinoma, microRNA-224-5p, PTEN, proliferation, invasion in vivo. PTEN reversed the effects of miR-224-5p on proliferation, migration and invasion of MCC1 cells. The present study revealed for the first time, to the best of the authors' knowledge, that miR-224-5p was highly expressed and served an oncogenic role in MCC. miR-224-5p not only regulated the proliferation, migration and invasion of pancreatic MCC but may also be a potential therapeutic target for MCC.

\section{Introduction}

Mucinous cystic neoplasms of the pancreas (MCNs) are a group of lesions that are usually benign but potentially malignant. They include mucinous cystadenoma (MCA), borderline mucinous cystadenoma (MCB) and mucinous cystadenocarcinoma (MCC). MCNs are common in women, with a male-female incidence rate of 1:9-1:20 (1-5). Pancreatic MCC is a rare malignant tumor, accounting for $<1 \%$ of pancreatic cancer types. In 1934, Lichtenstein (6) first reported a pancreatic MCC case that is notable due to it being a rare tumor and due to the complete clinical record in a period of 6 years may throw possible light on its pathogenesis. The case was an encapsulated cystic tumor of the tail of the pancreas, the size of a child's head and had in part undergone carcinomatous change after an interval of $\sim 5$ years, invading the capsule and metastasizing to the peritoneum, omentum and liver (6). Le Borgne et al (7) analyze 398 cases of cystadenomas of the pancreas between 1984 and 1996 in 73 institutions of the French Surgical Association in 1999. They identified $150 \mathrm{MCA}$ and $78 \mathrm{MCC}$ cases. In the 1970s, Compagno and Oertel (8) proposed that pancreatic MCA will eventually become malignant over time and indicate that the potential to invade is an innate intrinsic characteristic of pancreatic tumor exocrine cells rather than anacquired phenotype.

Non-coding RNAs, especially microRNAs (miRNAs/miRs), have gained attention due to their participation in numerous pathological processes, such as tumorigenesis, invasion and metastasis of tumors. miRNAs are an endogenous and small molecular non-coding RNA with a single strand. They can negatively regulate the target gene by combining with its 3'-untranslated region (3'-UTR) (9-11). Dynamic comparison of samples from different diseases in the progression of 
pancreatic cyst neoplasm, including the tumor tissue, peripheral blood and tumor cystic fluid, can reflect the pathogenesis of the malignant transformation of the pancreatic cells (12). Consistent with the WHO 2000 grade standard (13), the current research group used the expression profile chip of Agilent 16.0 to perform differential miRNA screening of the pathological tissues, cystic fluid and serum of patients with different diseases of MCN, including MCA, MCB and MCC. The present study anchored the key molecule miR-224-5p in miRNA by qPCR verification.

Although studies on miR-224-5p have received increasing attention (14-16), most of have been limited to common tumors. However, there area few studies on pancreatic MCC, which is rare in the clinic. In 1963, Cullen et al (17) searched 2.4 million medical records of Mayo Clinic and found only 17 patients who could be diagnosed with pancreatic cystadenocarcinoma. Thus, it is extremely difficult to conduct a study with fresh tissues of pancreatic MCC. The pancreatic disease group of Changhai Hospital is a national key discipline and $>500$ patients with pancreatic cancer are admitted annually. Paraffin specimens of MCC, which were surgically removed in Changhai hospital Affiliated to Naval Military Medical University between January 2012 and December 2016 were retrieved and only four cases were identified. In PubMed, there are few studies related to pancreatic MCC, most of which are case reports and the basic research related to pancreatic MCC was almost absent. Meanwhile, there is only one cell line of pancreatic MCC (MCC1 cell) globally.

Therefore, to further understand this rare tumor, more studies related to the functions and mechanisms of pancreatic MCC are required. To the best of the authors' knowledge, the present study is the first to systematically analyze the biological function and mechanism of miR-224-5p in pancreatic MCC in vitro and in vivo.

\section{Materials and methods}

Clinical samples. A total of four paired paraffin-embedded pancreatic MCC tumor samples and matched adjacent normal tissue samples were obtained from the Department of Pathology, Changhai Hospital (Shanghai, China). All cases were histologically confirmed. All four patients were female and the median age was 54.5 years (range, $47-76$ years). Lymph node metastasis was confirmed by pathology in three cases. In addition, the locations of the primary tumor in the patients were the tail and body of the pancreas, the tail in one patient and the body and tail in three patients. There was no history of radiotherapy or chemotherapy preoperatively. Informed consent was obtained from the subjects prior to specimen collection.

Cell culture. Human pancreatic ductal epithelial (HPDE) cells were obtained from the Shanghai Institute of Biochemistry and Cell Biology of the Chinese Academy of Sciences. The cells were cultured in DMEM (Thermo Fisher Scientific, Inc.) supplemented with 10\% FBS (HyClone; Cytiva) and $1 \%$ penicillin/streptomycin (Thermo Fisher Scientific, Inc.). MCC1 cells were provided by Professor Claudio Sorio from the University of Verona (Italy). They were incubated in RPMI-1640 medium (HyClone; Cytiva) supplemented with
$10 \% \mathrm{FBS}, 2 \mathrm{mM}$ glutamine, $80 \mu \mathrm{g} / \mathrm{ml}$ gentamicin sulphate and $2.5 \mu \mathrm{g} / \mathrm{ml}$ amphotericin B (Sigma-Aldrich; Merck KGaA). All cells were incubated at $37^{\circ} \mathrm{C}$ in a humidified atmosphere with $5 \% \mathrm{CO}_{2}$.

MTT assay. MTT assays wereperformed in 96-well plates (Corning, Inc.). The cells $\left(4 \times 10^{3}\right.$ cells $\left./ 100 \mu 1\right)$ were seeded for $24,48,72$ and $96 \mathrm{~h}$ at $37^{\circ} \mathrm{C}$ in a humidified atmosphere with $5 \% \mathrm{CO}_{2}$. After 24, 48, 72 and $96 \mathrm{~h}$ of culture, $10 \mu \mathrm{l}$ MTT (5 mg/ml, Sigma-Aldrich; Merck KGaA) solution was added to each well and cultured for another $4 \mathrm{~h}$. The culture medium was carefully removed and $100 \mu 1$ DMSO (Sigma-Aldrich; Merck KGaA) was added at room temperature for $30 \mathrm{~min}$. The absorbance value of each well was measured at $490 \mathrm{~nm}$ using a microplate reader (Bio-Rad Laboratories, Inc.).

Tumor xenografts. The animal experiments in the present study were performed in accordance with the protocol approved by the Institutional Animal Care and Use Committee of Shanghai Institute for Biological Sciences, Chinese Academy of Sciences (approval no. SIBS-2018-ZLX-2). In total, six 4-week-old female nude mice (BALB/c, nu/nu, weight, 20-25 g) were purchased from the Shanghai Experimental Animal Centre and maintained under pathogen-free conditions with $60-65 \%$ humidity at $22-25^{\circ} \mathrm{C}$ under a 12 -h light/dark cycle. All mice were allowed free access to drinking water and sterilized standard diet. Prior to the experiment, the nude mice were placed in pathogen-free conditions for 1 week. The nude mice were randomly divided into two groups with three in each group: Lenti-miR-224-5p-mimic group (Lenti-m) and Lenti-miR-224-5p-mimic negative control group (Lenti-c). On day 0 , tumor cells $\left(1 \times 10^{6}\right.$ cells/mouse $)$ suspended in $100 \mu \mathrm{l}$ serum-free medium and mixed 1:1 (v/v) with Matrigel were injected subcutaneously into the lower flank of each nude mouse. The tumor dimensions [length (L) and width $(\mathrm{W})]$ were measured twice a week for the tumor volume (volume $=\mathrm{W}^{2} \mathrm{x}$ L $\mathrm{x}$ 0.5). After 35 days frominoculation, the nude mice were sacrificed using cervical dislocation. Tumors were harvested, imaged, fixed and stored in liquid nitrogen.

Wound healing assay. The wound healing test was performed in 6 -well plates. Tumor cells $\left(3 \times 10^{5}\right.$ cells $\left./ 1,000 \mu \mathrm{l}\right)$ were seeded per well and incubated at $37^{\circ} \mathrm{C}$ in $5 \% \mathrm{CO}_{2}$. At $80 \%$ confluence, the scratches were created using a sterile $200 \mu \mathrm{l}$ pipette tip. Then, cells were washed gently to remove the floating cells and the medium was replaced with serum free medium for $24 \mathrm{~h}$. Images of the cells that had migrated into the wound were captured under an Olympus IX51 light microscope (Olympus Corporation; magnification, x10). The details of the wound healing assay were conducted as previously described (18-20).

Transwell migration and invasion assay. Transwell migration assay was performed in 24-well plates with $8-\mu \mathrm{m}$ pores (Corning, Inc.), whereas invasion assay was performed in 24-well plates with 8- $\mu \mathrm{m}$ pores coated with Matrigel (Corning, Inc.) according to the manufacturer's protocol. The tumor cells $\left(1 \times 10^{5}\right.$ cells/200 $\mu \mathrm{l}$ per well) were seeded into the upper chamber. The Matrigel was precoated at $37^{\circ} \mathrm{C}$ for $30 \mathrm{~min}$. In both the migration and invasion experiments, $500 \mu \mathrm{l}$ medium containing $10 \%$ FBS was added into the lower chamber as a 
Table I. Primer sequences used in reverse transcription-quantitative PCR.

\begin{tabular}{ll}
\hline Primer & \multicolumn{1}{c}{ Sequence $\left(5^{\prime} \rightarrow 3^{\prime}\right)$} \\
\hline miR-224-5p & F: CTCAACTGGTGTCGTGGAGTCGGCAATTCAGTTGAGCAAGGCAA \\
& R: ACACTCCAGCTGGGCAAGTCACTAGTGGT \\
U6 & F: CTCGCTTCGGCAGCACA \\
& R: AACGCTTCACGAATTTGCGT \\
PTEN & F: TGGATTCGACTTAGACTTGACCT \\
& R: GGTGGGTTATGGTCTTCAAAAGG \\
GAPDH & F: GGGGAGCCAAAAGGGTCATCATCT \\
& R: GACGCCTGCTTCACCACCTTCTTG
\end{tabular}

F, forward; $\mathrm{R}$, reverse; miR, microRNA.

chemoattractant. After $24 \mathrm{~h}$, the cells on the top surface of the Transwell chamber were removed using cotton swabs. The cells on the bottom surface were fixed at room temperature with $100 \%$ methanol for $30 \mathrm{~min}$, and then stained with $0.05 \%$ crystal violet for $30 \mathrm{~min}$ at room temperature. In total, five visual fields were randomly selected to be imaged with an Olympus IX51 light microscope (Olympus Corporation; magnification, $\mathrm{x} 20$ ). The details of the Transwell migration and invasion assay were conducted as previously described (18-20).

Target gene prediction and luciferase assay. The online tools TargetScanv7.2 (http://www.targetscan.org), PicTar version.2005 (https://pictar.mdc-berlin.de/cgi-bin/PicTar vertebrate.cgi) and miRanda version.2010 (http://www. microrna.org/microrna/getGeneForm.do) were used to predict miR-224-5p targets. The fragment of the PTEN 3'-UTR sequence containing one putative miR-224-5p binding site was cloned into the luciferase reporter plasmids (Shanghai GeneChem Co., Ltd.). GV369-miR-224 mimic-transfected MCC1 cells and GV369-miR-224 mimic-negative control-transfected MCC1 cells were transfected with the luciferase reporter using Lipofectamine ${ }^{\circledR} 2000$ (Invitrogen; Thermo Fisher Scientific, Inc.). A microplate reader was used to detect firefly luminescence and Renilla luminescence. Results were evaluated via normalization of the firefly luciferase activity with Renilla luciferase activity at $48 \mathrm{~h}$ post transfection using a Dual Luciferase Reporter assay (Promega Corporation). The details of the target gene prediction and luciferase assay were conducted as previously described (18-20).

Protein extraction and western blot assays. Cells were lysed with RIPA buffer (Cell Signaling Technology, Inc.) containing complete protease inhibitor cocktail (Roche Diagnostics), phosphatase inhibitors (Roche Diagnostics), $5 \mathrm{mM}$ dithiothreitol (Sigma-Aldrich; Merck KGaA) and $1 \mathrm{mM}$ phenylmethylsulfonyl fluoride (Sigma-Aldrich; Merck KGaA) and incubated on ice for $30 \mathrm{~min}$. The cell lysate was centrifuged at $12,000 \mathrm{x} \mathrm{g}$ for $10 \mathrm{~min}$ at $4^{\circ} \mathrm{C}$. The supernatant was collected and protein concentrations were determined using a BCA protein assay kit (Thermo Fisher Scientific, Inc.) according to the manufacturer's instructions. Western blot assays were performed as previously described (18). Then, $20 \mu \mathrm{g}$ protein was loaded onto a $10 \%$ gel, resolved using SDS-PAGE and transferred onto PVDF membranes. Membranes were then blocked with $5 \%$ fat-free milk for $2 \mathrm{~h}$ at room temperature. Subsequently, membranes were incubated with primary antibodies against PTEN (1:1,000; cat. no. 9552; Cell Signaling Technology, Inc.) and GAPDH (1:5,000; cat. no. M2006M; Abmart Pharmaceutical Technology Co., Ltd.) at $4^{\circ} \mathrm{C}$ overnight, washed three times with TBS-Tween-20 (TBST; 0.05\% Tween-20) and incubated with anti-mouse HRP-conjugated secondary antibody (1:2,000; cat. no. 7054S; Cell Signaling Technology, Inc.) at room temperature for $2 \mathrm{~h}$. Following three washes with TBST, immunoreactive bands were visualized using ECL working fluid (Biochannel; http: //www. biochannel.cn/page19. html?product_id=299).

$R N A$ extraction and reverse transcription-quantitative $P C R$ $(R T-q P C R)$. For RT-qPCR, TRIzol ${ }^{\circledR}$ reagent (Thermo Fisher Scientific, Inc.) was used to isolate the RNA of MCC-1 cells at $90 \%$ confluence, which was reverse transcribed into cDNA by Takara Prime Script RT kit (Takara Biotechnology Co., Ltd.) according to the manufacturer's instructions. The amount of cDNA was detected with a SYBR ${ }^{\circledR}$ Premix Ex Taq ${ }^{\mathrm{TM}}$ kit (Takara Biotechnology Co., Ltd.) in a StepOne Real-Time PCR Detection system (Thermo Fisher Scientific, Inc.). All expression data were normalized to U6-encoding transcript levels. RT-qPCR was performed as previously described (18). The total RNA was extracted from cells as aforementioned, and then miRNA was reverse transcribed and amplified using a Takara PrimeScript RT Reagent kit according to the manufacturer's instructions. The amplification reactions were performed in triplicate in a 96-well plate using the following thermocycling conditions for qPCR as follows: 5 min at $95^{\circ} \mathrm{C}$, followed by 40 cycles of $10 \mathrm{sec}$ at $95^{\circ} \mathrm{C}$ and $30 \mathrm{sec}$ at $60^{\circ} \mathrm{C}$. The $\mathrm{Cq}$ values were calculated using ABI Sequence Detection System software (version 2.1; Thermo Fisher Scientific, Inc.). Each samplewas analyzed in triplicate and levels were quantified using the $2^{\Delta \Delta \mathrm{Cq}}$ method (21). The primers used for RT-qPCR are presented in Table I.

Small interfering (si)RNA and cell transfection. siRNA was purchased from Guangzhou RiboBio Co., Ltd. The sequence of PTEN siRNA was 5'-ACCAGGACCAGAGGAAACCT-3' and 
negative control siRNA (siRNA NC) was 5'-AUUGGCUACU ACCGAAGAG-3'. pcDNA vector expression PTEN and empty pcDNA vector were freely provided by Dr Renxu Chang (Shanghai Institute of Nutrition and Health, Shanghai Institutes for Biological Sciences). The siRNA was co-transfected in miR-224-5p inhibitor and NC MCC1 cells $\left(5 \times 10^{4}\right)$ and pcDNA vector were co-transfected in miR-224-5p mimic and negative control MCC1 cells $\left(5 \times 10^{4}\right)$, using Lipofectamine ${ }^{\circledR} 2000$ reagent (Thermo Fisher Scientific, Inc.) according to the manufacturer's protocol; the siRNA and Lipofectamine ${ }^{\circledR} 2000$ was mixed at room temperature and was added to the cells after $25 \mathrm{~min}$. The ratio of siRNA to Lipofectamine ${ }^{\circledR} 2000$ was $1 \mu \mathrm{g}: 2 \mu \mathrm{l}$. Cells were transfected for $48 \mathrm{~h}$ before performing subsequent experiments.

Vectors and lentiviral transduction. All recombinant lentiviruses were obtained from Shanghai Genechem Co., Ltd. The packaged lentiviruses contained miR-224-5p mimic, miR-224-5p inhibitor and miR-224-5p mimic control, miR-224-5p inhibitor and miR-224-5p inhibitor control. All negative controls were empty vectors. Lentiviral vector of miR-224-5p mimic and miR-224-5p inhibitor was GV280 and GV390, respectively. The sequences were as follows: hsa-miR-224-5p-mimic forward, 5'-GAGGATCCCCGGGT ACCGGCCAGCTAACCATGGGCCTGCCTC-3' and reverse, 5'-CACACATTCCACAGGCTAGAGGAGAAAGAAGACCT CTTTTC-3', hsa-miR-224-5p-inhibitor forward,5'-CAAGTCA CTAGTGGTTCCGTT-3' and reverse, 5'-AACGGAACCAC TAGTGACTTG-3'. Lentiviral transduction was performed according to the manufacturer's protocol. The packaged virus solution was thawed and the virus stock solution was diluted with the infection medium value $=10 \mathrm{using}$ fresh medium containing the gene transfection enhancer polyamine. After $72 \mathrm{~h}$ of infection at room temperature, the successes transduction was observed by green fluorescent protein-positive cells observed under an inverted fluorescence microscope (magnification, x200; Olympus Corporation). The lentivirus-infected cells were treated with $2 \mu \mathrm{g} / \mathrm{ml}$ puromycin for 1 week and the cells resistant to puromycin were selected. Then, the expression of miR-224-5p in the groups of Lent-c, Lent-m and Lent-i were quantified by qPCR.

Immunohistochemistry (IHC). IHC analysis was performed as previously described (18). Deparaffinized and rehydrated sections were incubated with $3 \% \mathrm{H}_{2} \mathrm{O}_{2}$ in methanol for $10 \mathrm{~min}$ at room temperature to block endogenous peroxidase activity. The sections were then subjected to antigen retrieval for $10 \mathrm{~min}$ in a pressure cooker containing sodium citrate, and were incubated in 5\% normal goat serum (cat.no. DXT 50197Z; Invitrogen; Thermo Fisher Scientific, Inc.) for $20 \mathrm{~min}$ at $37^{\circ} \mathrm{C}$, permeabilized in PBS Triton solution and incubated with a primary antibody against PTEN (1:200; cat. no. 9552; Cell Signaling Technology, Inc.) at $4^{\circ} \mathrm{C}$ overnight. The sections were then incubated with anti-mouse HRP-conjugated secondary antibody (1:1,000; cat. no. 7054S; Cell Signaling Technology, Inc.) at $37^{\circ} \mathrm{C}$ for $1 \mathrm{~h}$. The sections were then counterstained with hematoxylin for $5 \mathrm{~min}$ at room temperature, and finally dehydrated and covered with a coverslip. The sections were observed under an IX51 light microscope (Olympus Corporation; magnification, x40). Each experiment was performed three times. IHC evaluation of protein expression intensity in normal adjacent tissues and paired pancreatic MCC tissues was performed independently by two pathologists from the Department of Pathology, Changhai Hospital. Staining intensity was scored as previously described (22).

Statistical analysis. Data are presented as mean \pm standard deviation from at least three independent experiments. Student's t-test was used to compare the differences between the two groups; one-way ANOVA followed by Tukey's post hoc test was used to compare the differences among multiple groups. SPSS 21.0 software (IBM Corp.) was used to conduct statistical analysis. The experimental images were edited and processed using Photoshop 19.0 (Adobe Systems, Inc.) and GraphPad Prism 5.0 (GraphPad Software, Inc.). $\mathrm{P}<0.05$ was considered to indicate a statistically significant difference.

\section{Results}

Effect of miR-224-5p on the proliferation of MCC1 cells in vitro and in vivo. The results demonstrated that the expression of miR-224-5p in MCC1 cells was significantly higher compared with HPDE cells (Fig. 1A). Lentivirus with different expression of miR-224-5p (high, low and negative control) was used to transfect MCC1 cells. A total of three groups of MCC1 cells: Lenti-miR-224-mimic group (Lenti-m), lenti-miR-224 inhibitor group (Lenti-i) and Lenti-miR-224 control group (Lenti-c) were obtained. Following puromycin screening, MCC1 cells with stably different expression of miR-224-5p were obtained (Fig. 1C). Fig. 1C (left) illustrates the cells resistant to puromycinasselected under an inverted fluorescence microscope. Fig. 1C (right) illustrates the expression of miR-224-5p in the groups of Lent-c, Lent-m and Lent-i from qPCR.

MTT was then used to measure the effect of miR-224-5p on the proliferation of MCC1 cells. The results demonstrated that the proliferation ability of $\mathrm{MCC} 1$ cells in the Lenti-m group was significantly higher compared withthe Lenti-c group after $48 \mathrm{~h}$ and the difference was more pronounced after $72 \mathrm{~h}$, while the proliferation activity of MCC1 cells in the Lenti-i group was significantly lower compared with the Lenti-c group after $72 \mathrm{~h}$ (Fig. 1B). To further observe the effects of miR-224-5p on the promotion of the proliferation of MCC1 cells in vivo, a tumorigenesis assay in nude mice was performed using MCC1 cells with stable miR-224-5p overexpression. The authors of the present study were the first to report MCC subcutaneous tumors in nude mice. The tumors were cystic with white fluid (Fig. 1D). The tumor dimensions ( $\mathrm{L}$ and $\mathrm{W}$ ) were measured twice a week for the tumor volume (volume $=\mathrm{W}^{2} \times \mathrm{L} \times 0.5$ ). The tumors in the Lenti-m group grew faster than those in the Lenti-cgroup (Fig. 1E). The tumors in the Lenti-m group were larger than those in the stable transfer Lenti-cgroup (Fig. 1F). RT-qPCR was used to detect miR-224-5p expression in the tumor tissues. The results verified that miR-224-5p expression in the Lenti-m group was higher compared with the Lenti-c group in vivo (Fig. 1G).

Effect of miR-224-5p on the migration and invasion of MCCI cells. Wound healing assay and Transwell assay were used to detect cell migration and invasion of MCC1 cells. miR-224-5p 
A

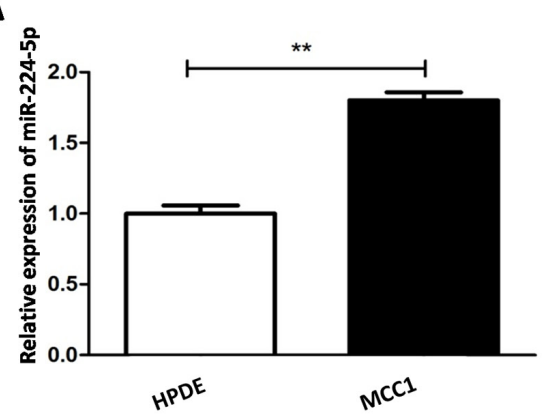

C

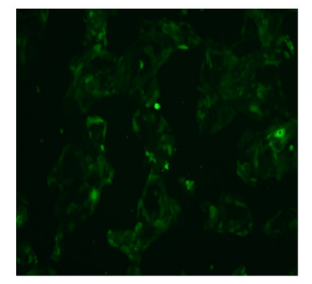

Lenti-c

D

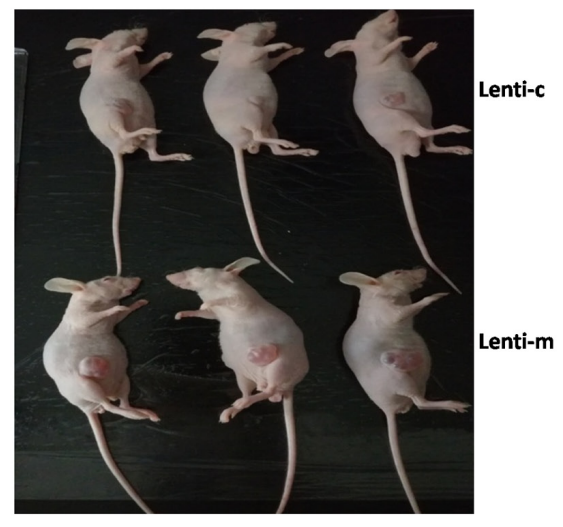

F

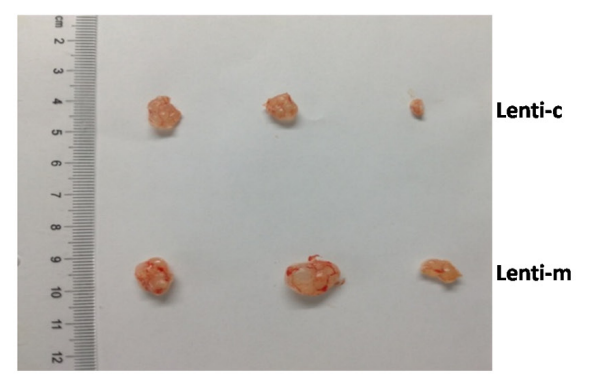

B
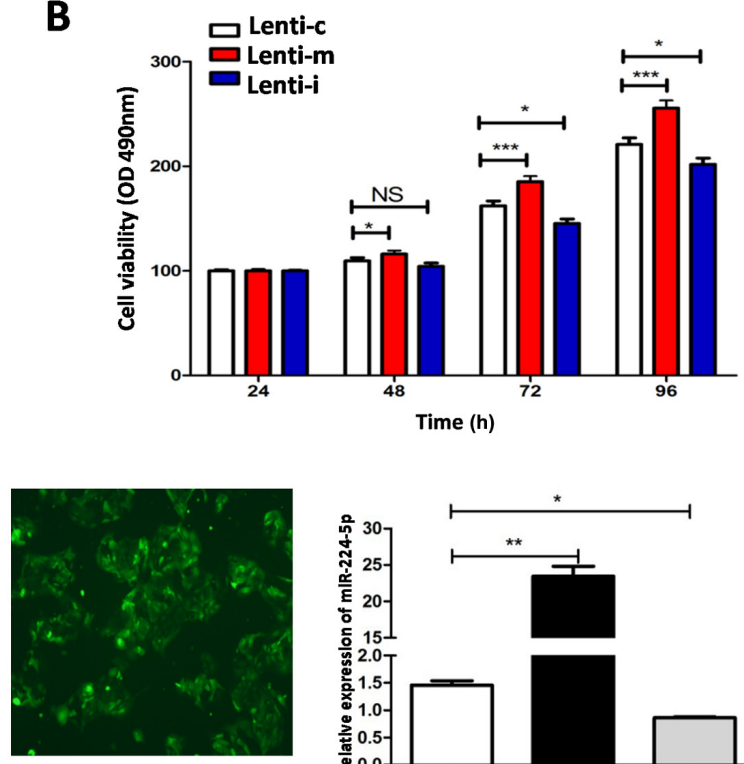

enentiti

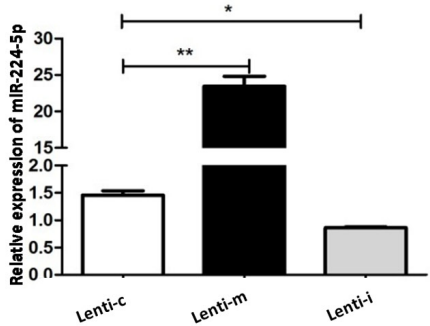

E

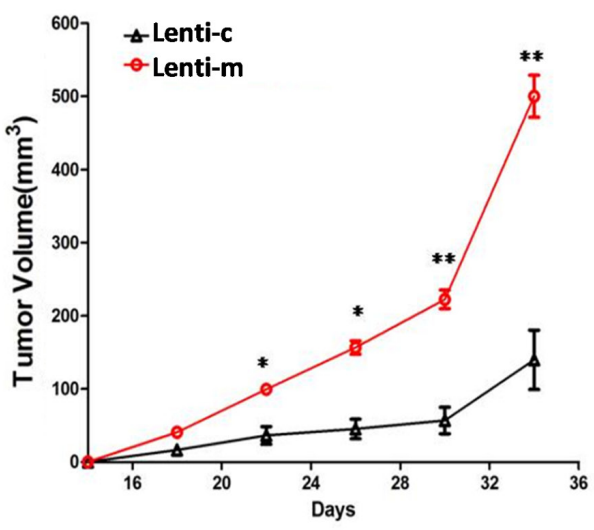

G

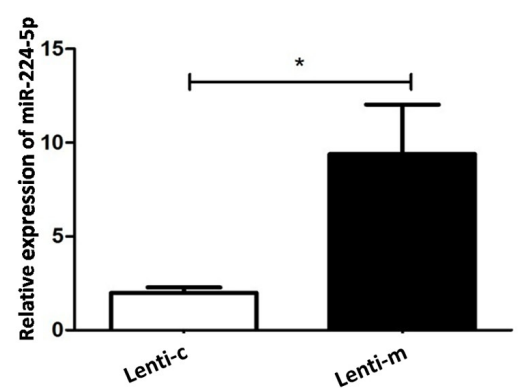

Figure 1. Effect of miR-224-5p on the proliferation of MCC1 cells. (A) Expression of miR-224-5p in mucinous cystadenocarcinoma and HPDE cells. (B) Effect of miR-224-5p on the proliferation of MCC1 cells in vitro. (C) MCC1 cells transfected with miR-224-5p lentivirus vector and validation of the expression of miR-224-5p in these cells: Left illustrates the cells resistant to puromycin under an inverted fluorescence microscope; right illustrates the expression of miR-224-5p in the groups of Lenti-c, Lenti-m and Lenti-i from reverse transcription-quantitative PCR. (D-G) Effect of miR-224-5p on the proliferation of MCC1 cells in vivo. (D) Appearance of the tumor in mice. (E) Curve of tumor growth. (F) Tumors. (G) Expression of miR-224-5p in the tumors of nude mice. ${ }^{*} \mathrm{P}<0.05,{ }^{* *} \mathrm{P}<0.01,{ }^{* * *} \mathrm{P}<0.001$. miR, microRNA; MCC, mucinous cystadenocarcinoma; HPDE, human pancreatic ductal epithelial; Lent-c, lenti-miR-224-5p-mimic negative control group; Lent-m, lenti-miR-224-5p-mimic group; Lent-I,lenti-miR-224 inhibitor group.

overexpression promoted the proliferation and migration abilities of MCC1 cells and knockdown of miR-224-5p expression inhibited the proliferation and migration abilities of $\mathrm{MCC} 1$ cells in vitro (Fig. 2A-C).

PTEN is the target gene of miR-224-5p. The potential target genes of miR-224-5p that regulate proliferation and invasion were screened using the bioinformatics algorithm TargetScan Human 7.2. There was a potential binding site of 6 bp between miR-224-5p and the PTEN gene, which was selected for experimental validation (Fig. 3A). To confirm whether PTEN was a direct target of miR-224-5p, luciferase reporter assay was performed. The results demonstrated that miR-224-5p overexpression reduced luciferase activity in the PTEN 

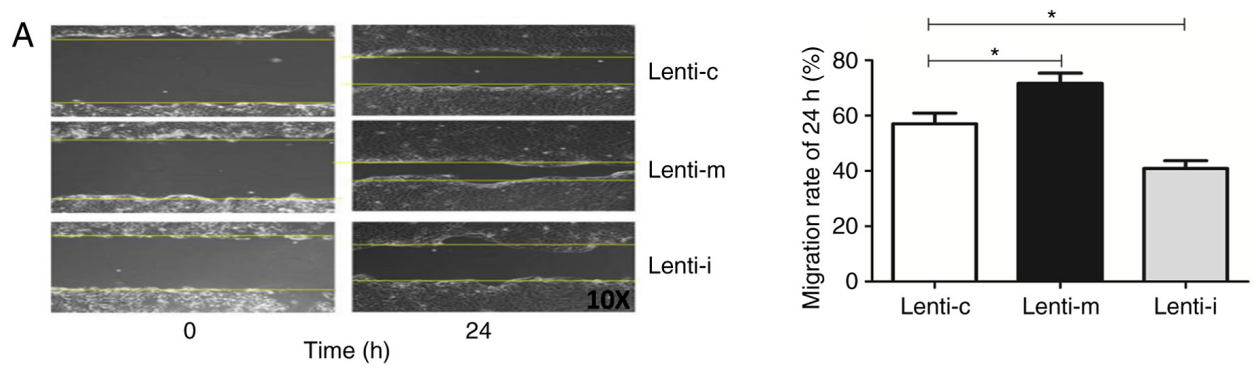

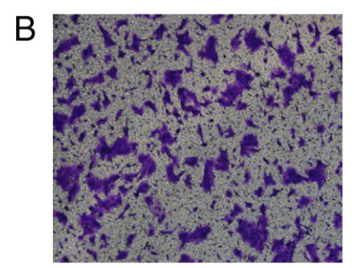

Lenti-c

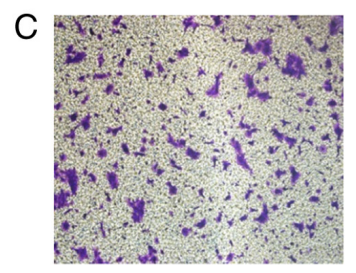

Lenti-c

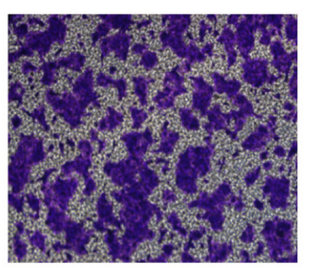

Lenti-m

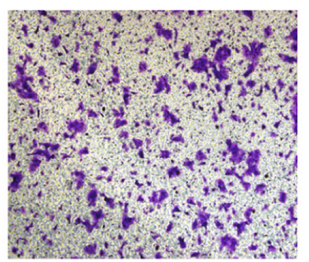

Lenti-m

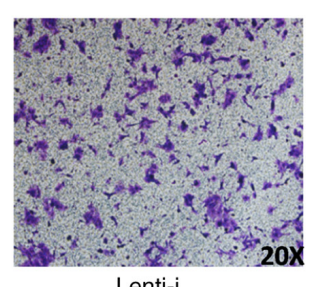

Lenti-i

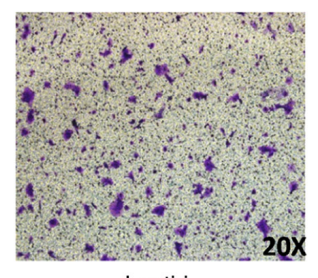

Lenti-i
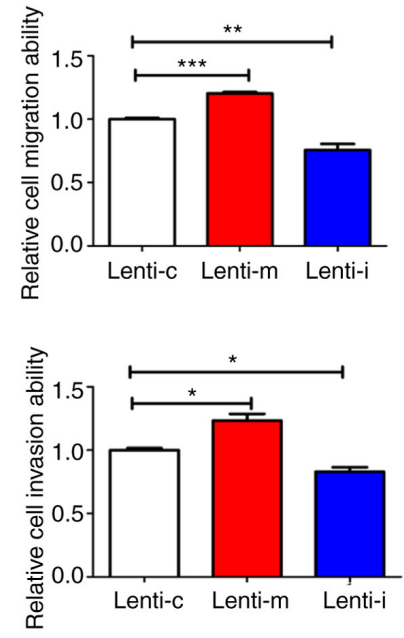

Figure 2. Effect of miR-224-5p on the migration and invasion of MCC1 cells. (A) Effect of miR-224-5p on the migration of MCC1 cells by the wound healing assay (magnification, x10). (B) Effect of miR-224-5p on the migration of MCC1 cells by Transwell migration assay (magnification, x10). (C) Effect of miR-224-5p on the invasion of MCC1 cells by Transwell assay (magnification, $\mathrm{x} 10$ ). ${ }^{*} \mathrm{P}<0.05,{ }^{* *} \mathrm{P}<0.01,{ }^{* * * *} \mathrm{P}<0.001$. miR, microRNA; MCC, mucinous cystadenocarcinoma; Lent-c, lenti-miR-224-5p-mimic negative control group; Lent-m, lenti-miR-224-5p-mimic group; Lent-I, lenti-miR-224 inhibitor group.

A

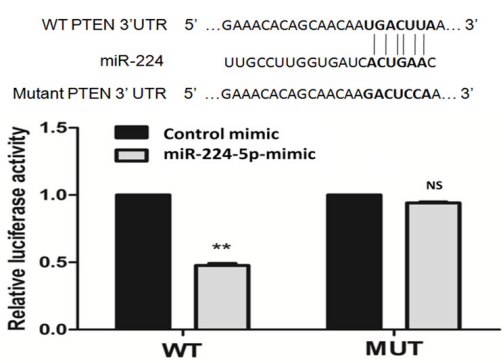

B

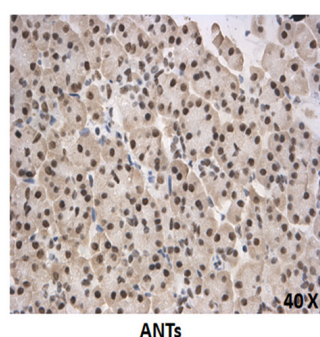

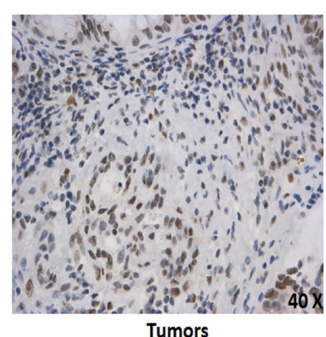

Tumors
C

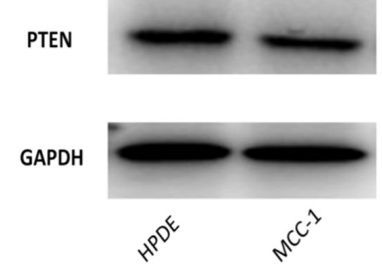

D

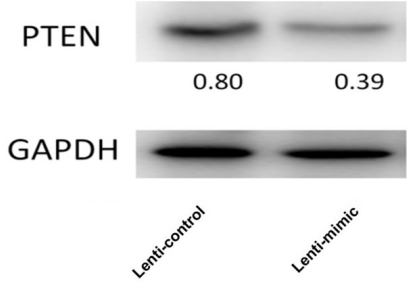

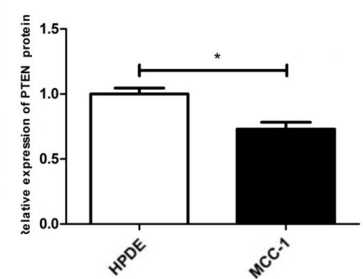
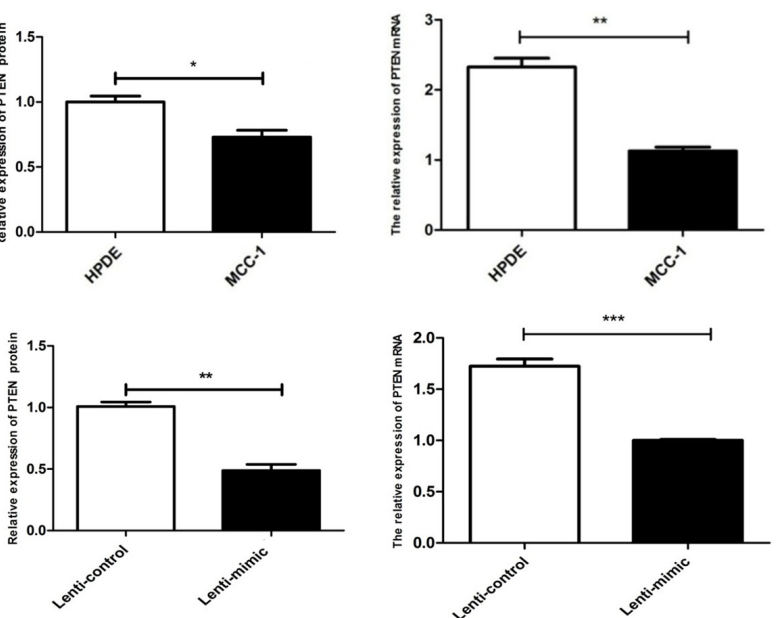

Figure 3. PTEN is the target gene of miR-224-5p. (A) Luciferase report on miR-224-5p and PTEN target gene. (B) Expression of PTEN in pancreatic MCC tissues and matched adjacent normal tissues (magnification, $\mathrm{x} 40$ ). (C) Expression of PTEN in MCC1 and HPDE cells. (D) Protein and mRNA expression level of PTEN in MCC1 cells in vivo. ${ }^{*} \mathrm{P}<0.05,{ }^{* *} \mathrm{P}<0.01,{ }^{* * *} \mathrm{P}<0.001$. NS, $\mathrm{P}>0.05$. miR, microRNA; MCC, mucinous cystadenocarcinoma; HPDE, human pancreatic ductal epithelial; WT, wild-type; MUT, mutation type; ANTs, adjacent normal tissues. 
A

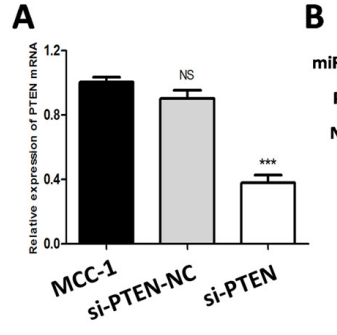

D

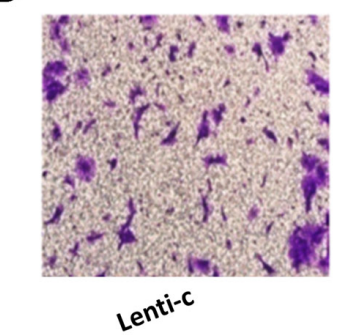

E

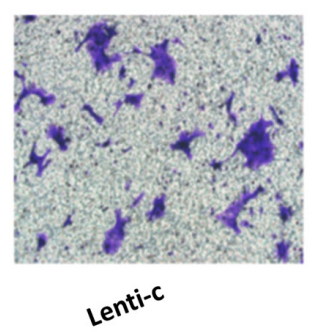

B

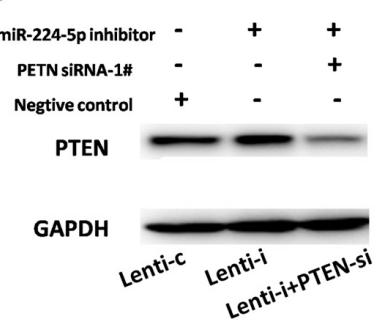

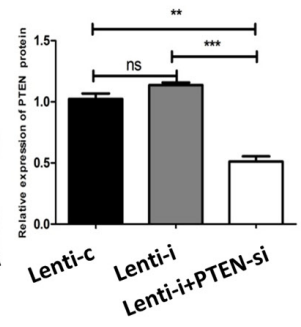

C
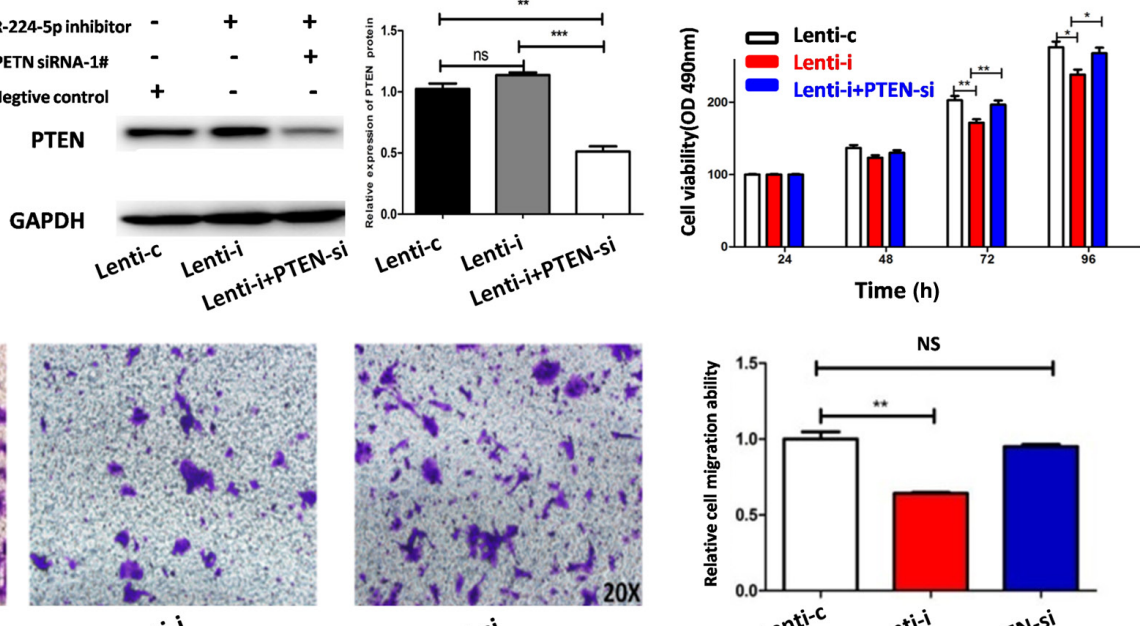

Lentiti

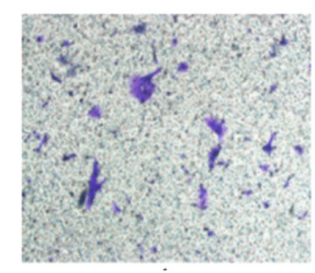

Lenti-i
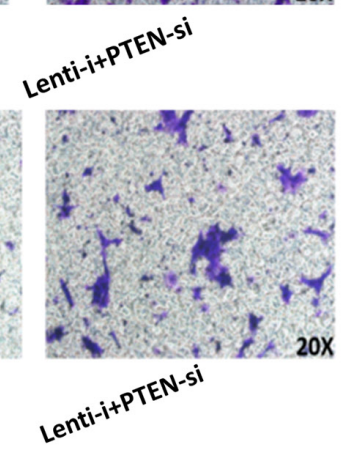
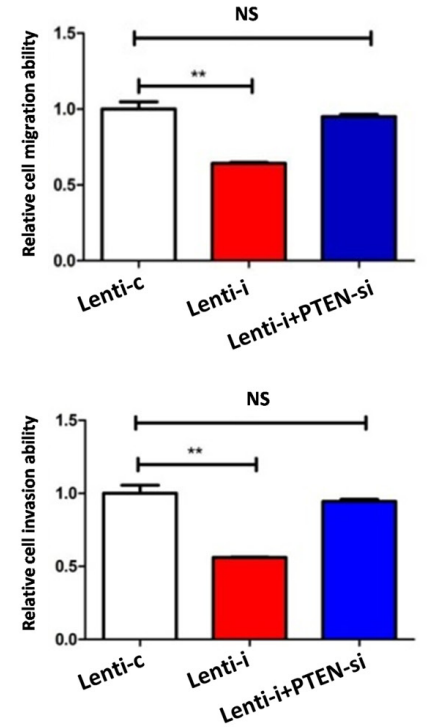

Figure 4. Inhibiting PTEN expression in MCC1 cells reverses the inhibition of proliferation, migration and invasion induced by low miR-224-5p expression. (A) The transfections of si-PTEN were verified as successful by PCR. (B) Expression of PTEN in MCC1 cells of Lenti-c, Lenti-i and Lenti-i+PTENsi group. Inhibition of PTEN expression in MCC1 cells reverses the inhibition of (C) viability, (D) migration and (E) invasion induced by low miR-224-5p expression. ${ }^{*} \mathrm{P}<0.05,{ }^{* * *} \mathrm{P}<0.01,{ }^{* * * *} \mathrm{P}<0.001$. NS, $\mathrm{P}>0.05$. MCC, mucinous cystadenocarcinoma; miR, microRNA; si, small interfering; Lent-c, lenti-miR-224-5p-mimic negative control group; Lent-m, lenti-miR-224-5p-mimic group; Lent-i, lenti-miR-224 inhibitor group; Lenti-i+PTENsi, Lenti-i+PTEN siRNA-1\#.

wild-type (WT) 3'-UTR reporter but had no effect on the PTEN mutant (MUT) 3'-UTR reporter (Fig. 3A). To further confirm that PTEN was a target of miR-224-5p, the mRNA and protein expression of PTEN was examined using RT-qPCR and western blot analysis. The results demonstrated that PTEN mRNA and protein levels were significantly downregulated in normal $\mathrm{MCC} 1$ cells and xenograft tumors transfected with miR-224-5p-overexpressing MCC1 cells, whereas the expression of these levels was upregulated in HPDE cells and xenograft tumors transfected with miR-224-5p-overexpressing-control MCC1 cells (Fig. 3C and D). miR-224-5p expression was negatively associated with PTEN in MCC1 cells in vitro (Figs. $1 \mathrm{~A}$ and $3 \mathrm{C}$ ) and in vivo (Figs. $1 \mathrm{G}$ and $3 \mathrm{D}$ ). These findings indicated that PTEN was a target of miR-224-5p in $\mathrm{MCC} 1$ cells. The expression of PTEN protein in the tumor and matched adjacent normal tissues was detected by IHC. The results demonstrated that the expression of PTEN protein in the tumor was lower compared with matched adjacent normal tissues (Fig. 3B).

miR-224-5p regulates the proliferation, migration and invasion of MCCl cells by targeting PTEN. The transfection of si-PTEN to MCC1 cells was successful (Fig. 4A). The miR-224-5p-inhibitor, PTEN siRNA, or negative control was transfected into MCC1 cells to investigate the effect of PTEN on MCC1 cells. The expression of PTEN was detected by western blot analysis (Fig. 4B). MTT and Transwell assays were used to evaluate the effect on proliferation, migration and invasion in MCC1 cells. The results demonstrated that the proliferation, migration and invasion of MCC1 cells transfected with Lenti-miR-224-5p inhibitor decreased compared to those of the MCC1 cells transfected with the negative control. The effect of PTEN on proliferation, migration and invasion in $\mathrm{MCC} 1$ cells downregulated by miR-224-5p were then investigated. It was found that inhibiting PTEN expression could reverse the inhibitory effect of miR-224-5p on MCC1 cells (Fig. 4C-E) after which the transfection of PTEN plasmid into the MCC1 cells was successful (Fig. 5A). miR-224-5p mimic, PTEN plasmid or $\mathrm{NC}$ was also transfected into $\mathrm{MCC1}$ cells to investigate the effect of PTEN on MCC1 cells. PTEN expression was detected by western blot analysis (Fig. 5B). MTT and Transwell assays were used to test the effect of proliferation, migration and invasionin MCC1 cells. The results demonstrated that the proliferation, migration and invasion of MCC1 cells transfected with Lenti-miR-224-5p mimic increased compared to those in $\mathrm{MCC} 1$ cells transfected with the NC. Then, the effect of PTEN on proliferation, migration and invasion in $\mathrm{MCC} 1$ cells upregulated by miR-224-5p were investigated. It was found that increasing PTEN expression could reverse the inhibitory effect of miR-224-5p on MCC1 cells (Fig. 5C-E). 
A
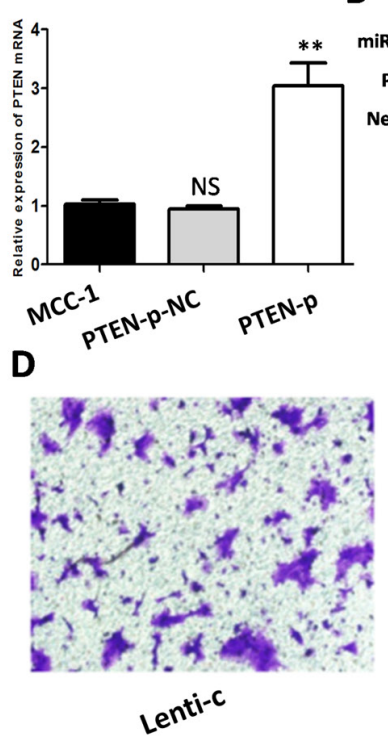

E

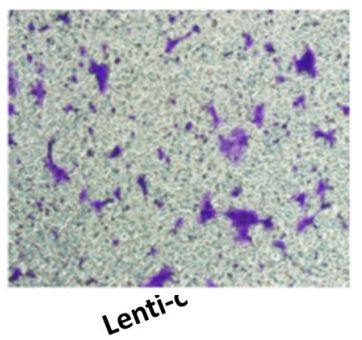

B
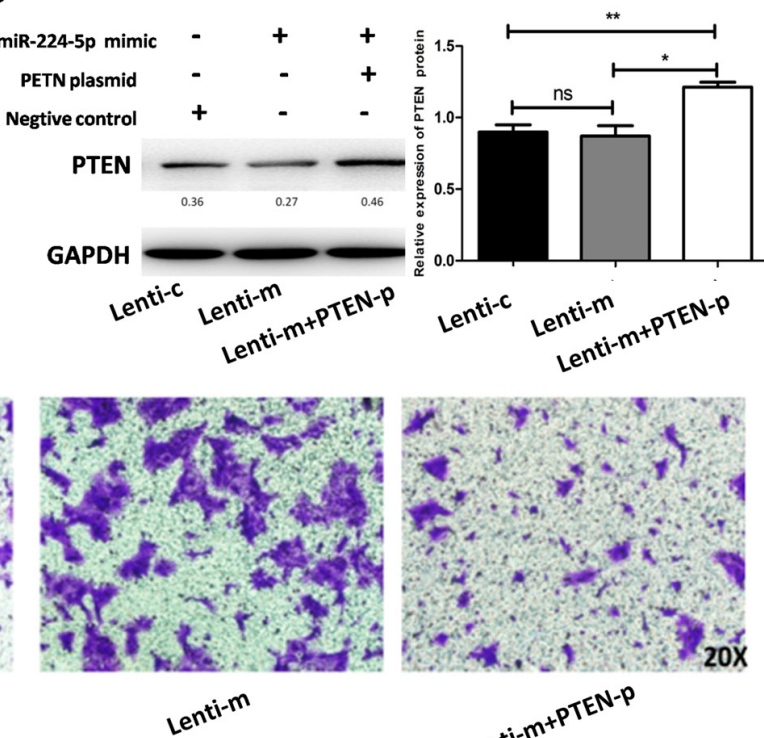

Lenti-m
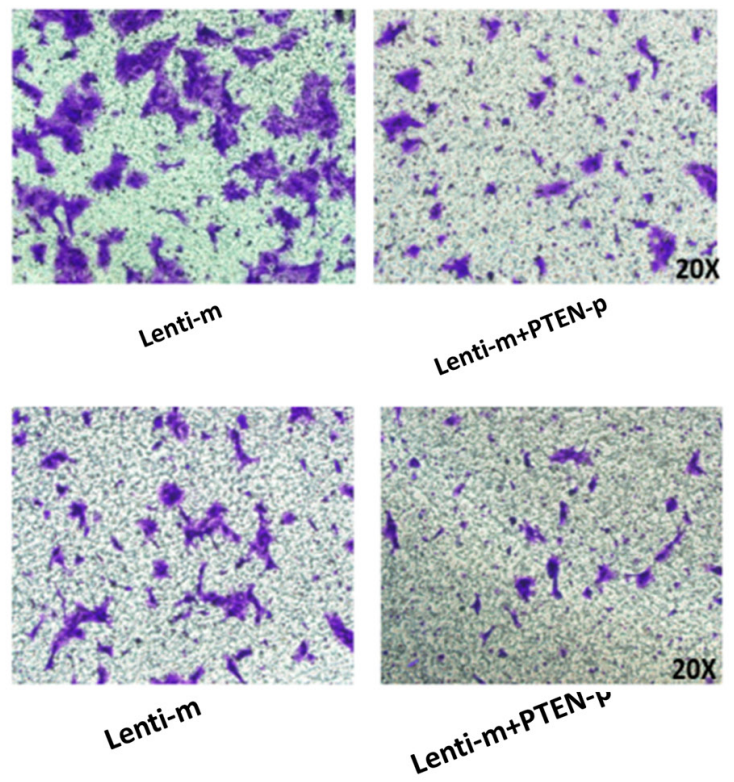

C
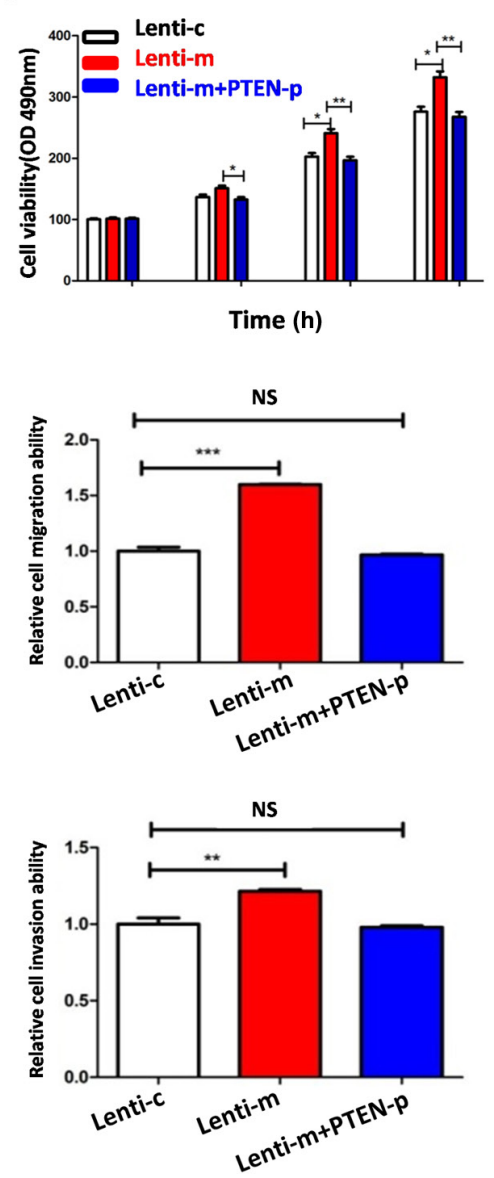

Figure 5. Increasing PTEN expression in MCC1 cells reverses the promotion of proliferation, migration and invasion induced by miR-224-5p overexpression. (A) The transfections of PTEN plasmid were verified as successful by PCR. (B) Expression of PTEN in MCC1 cells of Lenti-c, Lenti-m and Lenti-m+PTEN-p groups. Increasing PTEN expression in MCC1 cells reverse the promotion of (C) viability, (D) migration and (E) invasion induced by miR-224-5p overexpression. ${ }^{*} \mathrm{P}<0.05,{ }^{* *} \mathrm{P}<0.01,{ }^{* * * *} \mathrm{P}<0.001$. NS, $\mathrm{P}>0.05$. MCC, mucinous cystadenocarcinoma; miR, microRNA; Lent-c, lenti-miR-224-5p-mimic negative control group; Lent-m, lenti-miR-224-5p-mimic group; Lent-i, lenti-miR-224 inhibitor group; Lenti-m+PTENp, Lenti-m+PTEN plasmid.

\section{Discussion}

Abnormal proliferation is a unique feature of tumor cells andthe inhibition of tumor cell proliferation is controversial (23). miRNA serves an important role in tumorigenesis and the development of malignant tumors and regulates the proliferation and invasion of tumor cells by combining with target genes (24-26).

Pancreatic MCC is a rare malignant tumor, with a limited number of studies, to the best of the authors' knowledge, and there is only one cell line of pancreatic MCC (MCC1) worldwide. Therefore, to further understand this rare tumor, more studies on the functions and mechanisms of pancreatic MCC are urgently required. We had previously established and verified the key molecule miR-224-5p in pancreatic MCNs (27). The present study found that miR-224-5p expression in MCC1 cells was significantly higher compared with HPDE cells, indicating that miR-224-5p might serve an oncogenic role in pancreatic MCC. It was observed that miR-224-5p overexpression in $\mathrm{MCC} 1$ cells promoted proliferation, migration and invasion, while low miR-224-5p expression in MCC1 cells significantly inhibited proliferation, migration and invasion. This is consistent with previously reported studies (28-30). The present study also assessed the effect of miR-224-5p on
MCC1 cells in nude mice. To the best of the authors' knowledge, this is the first study to construct subcutaneous tumors in pancreatic MCC in vivo. The tumors that were cystic and contained white fluid were observed at 10-14 days in all nude mice. These characteristics of tumors in mice were similar to the pathological characteristics of patients diagnosed with pancreatic MCC in the clinic. The present study verified that miR-224-5p overexpression in MCC1 cells promoted proliferation in nude mice. To the best of the authors' knowledge, the present study was the first on pancreatic MCC in nude mice. miRNAs can regulate the biological function of cells by binding with the 3'-UTR of the target gene (31). Bioinformatics software, including miRanda, TargetScan and PicTar, is commonly used to predict miRNA target genes $(32,33)$. The three databases (TargetScan, PicTar and miRanda) were used to explore the target genes of miR-224-5p. Combined with the research reported in the literature, the PTEN gene was screened, which is related to tumor proliferation and invasion (34). The present study verified the targeting relationship between miR-224-5p and PTEN using a luciferase reporter assay, which provided direct evidence of miR-224-5p targeting the PTEN gene. Simultaneously, RT-qPCR and western blot analysis were used to further confirm that miR-224-5p expression was negatively associated with PTEN in MCC1 cells in vitro and 
in vivo. As pancreatic MCC is rare in the clinic, it is extremely difficult to detect miR-224-5p expression in fresh tissues of pancreatic MCC and miR-224-5p was degraded in the paraffin tissue of pancreatic MCC. Therefore, PTEN protein was only detected in pancreatic MCC paraffin-embedded samples and it was found that PTEN protein expression in tumors was lower compared with adjacent normal tissues. Therefore, these results directly and indirectly support the finding that PTEN is the target gene of miR-224-5p in MCC1 cells.

PTEN, a tumor suppressor gene, mainly regulates the PI3K/AKT, MAPK and focal adhesion kinase signaling pathways and serves an important role in regulating cell proliferation, apoptosis, cell signal transduction, tumor cell infiltration, metastasis, drug resistance and angiogenesis (35-37). miRNAs serve a role in the inactivation of PTEN. In breast cancer tissues and cells, miR-182-5p is highly expressed and patients with breast cancer with high miR-182-5p expression are associated with a low survival rate. Knocking down miR-182-5p expression in breast cancer cells can inhibit the proliferation and invasion of breast cancer cells and PTEN is considered the target of miR-182-5p. The recovery of PTEN expression can reverse the miR-182-5p-mediated promotion of breast cancer cell proliferation and invasion (38). In hepatocellular carcinoma, Jiang et al (39) found that miR-19a-3p promoted tumor metastasis and chemoresistance through the PTEN/AKT pathway.

To clarify that miR-224-5p mediates the proliferation, migration and invasion of pancreatic MCC by targeting PTEN, PTEN siRNA and plasmids were used to regulate the expression of PTEN in MCC1 cells. The present study found that low miR-224-5p expression could inhibit the proliferation, migration and invasion of MCC1 cells, while PTEN inhibition could reverse the biological effect of low miR-224-5p expression in MCC1 cells. Meanwhile, miR-224-5p overexpression in MCC1 cells promoted proliferation, migration and invasion, while PTEN overexpression reversed the biological effect of miR-224-5p overexpression in MCC1 cells. These results confirmed that miR-224-5p partly mediates the proliferation, migration and invasion of MCC1 cells by regulating PTEN.

Tumorigenesis and the development of pancreatic MCC involve many factors, including genes, proteins andmolecules (40). The present study was the first, to the best of the authors' knowledge, to systematically evaluate the relationship between miR-224-5p and the PTEN gene in vitro and in nude mice and clinical tissue samples. It was confirmed that miR-224-5p can regulate the proliferation, migration and invasion of pancreatic MCC by targeting PTEN. These results suggested that $\mathrm{miR}-224-5 \mathrm{p}$ serves an oncogenic role in MCC. The present study analyzed the relationship between miR-224-5p and pancreatic MCC with respect to miRNA. The present study enriched the basic and clinical knowledge on pancreatic MCC. It is hoped that the present study will bring breakthroughs in the targeted and precise treatment of pancreatic MCC in the future.

There were some limitationsin the present study. First, the number of patients was too small (only four patients). Second, there was only one pancreatic MCC cell line (MCC1) worldwide. Third, the study only verified that miR-224-5p overexpression in $\mathrm{MCC} 1$ cells promoted proliferation in nude mice in vivo. Last, as pancreatic MCC is rare in the clinicand
miR-224-5p was degraded in paraffin tissue of pancreatic MCC, the present study only detected PTEN protein in pancreatic MCC paraffin-embedded samples. Some of these concerns are worthy of further study.

\section{Acknowledgements}

The authors would like to thank Professor Claudio Sorio (University of Verona, Italy) for the MCC1 cell line.

\section{Funding}

The present study was supported by grants from the National Natural Science Foundation of China (grant nos. 81672892 and 82072707) and the Shanghai Anticancer Association EYAS PROJECT (grant no. SACA-CY1C12).

\section{Availability of data and materials}

The datasets used and/or analyzed during the current study are available from the corresponding author on reasonable request.

\section{Authors' contributions}

$\mathrm{XZ}$ and LZ conceived the study. XP, CG, LS and YW acquired the data. LS and MY analyzed the data. XZ acquired funding. $\mathrm{XP}, \mathrm{CG}, \mathrm{YW}$ and RC performed the experiments. RC and MY developed the methodology. XZ and MY were responsible for project administration. XP, CG and YW interpreted the results. $\mathrm{XP}$ wrote the manuscript. $\mathrm{XZ}$ and $\mathrm{LZ}$ revised the manuscript. $\mathrm{XP}, \mathrm{CG}$ and $\mathrm{YW}$ confirmed the authenticity of all the raw data. All authors have reviewed and approved the final version of the manuscript.

\section{Ethics approval and consent to participate}

The present study was approved by the Ethics Committee of the Changhai Hospital (approval no. CHEC-2016 8167111578). The animal experiments was approved by the Institutional Animal Care and Use Committee of Shanghai Institute for Biological Sciences, Chinese Academy of Sciences (approval no. SIBS-2018-ZLX-2).

\section{Patient consent for publication}

Not applicable.

\section{Competing interests}

The authors declare that they have no competing interests.

\section{References}

1. Brewer Gutierrez OI and Lennon AM: Pancreatic Cysts: Sinister Findings or Incidentalomas? Med Clin North Am 103: 163-172, 2019.

2. Dorobanţu BM, Matei E, Herlea V, Boroş M, Tivadar B and Ciurea SH: Diagnosis, morphopathological profile and treatment of mucinous cystadenoma of the pancreas - a single center experience. Rom J Morphol Embryol 59: 1155-1163, 2018.

3. Becker WF, Welsh RA and Pratt HS: Cystadenoma and cystadenocarcinoma of the pancreas. Ann Surg 161: 845-863, 1965. 
4. Katoh H, Rossi RL, Braasch JW, Munson JL, Shimozawa E and Tanabe T: Cystadenoma and cystadenocarcinoma of the pancreas. Hepatogastroenterology 36: 424-430, 1989.

5. Doulamis IP, Mylonas KS, Kalfountzos CE, Mou D, Haj-Ibrahim H and Nasioudis D: Pancreatic mucinous cystadenocarcinoma: Epidemiology and outcomes. Int J Surg 35: 76-82, 2016.

6. Lichtenstein L: Papillarycystadenocarcinoma of pancreas. case report, with notes on classification of malignant cystic tumors of pancreas. Am J Cancer 21: 542-553, 1934.

7. Le Borgne J, de Calan L and Partensky C; French Surgical Association: Cystadenomas and cystadenocarcinomas of the pancreas: A multiinstitutional retrospective study of 398 cases. Ann Surg 230: 152-161, 1999.

8. Compagno J and Oertel JE: Mucinous cystic neoplasms of the pancreas with overt and latent malignancy (cystadenocarcinoma and cystadenoma). A clinicopathologic study of 41 cases. Am J Clin Pathol 69: 573-580, 1978

9. Lu J, Getz G, Miska EA, Alvarez-Saavedra E, Lamb J, Peck D, Sweet-Cordero A, Ebert BL, Mak RH, Ferrando AA, et al: MicroRNA expression profiles classify human cancers. Nature 435: 834-838, 2005.

10. Van Roosbroeck K and Calin GA: Cancer Hallmarks and MicroRNAs: The Therapeutic Connection. Adv Cancer Res 135 119-149, 2017.

11. Rawat M, Kadian K, Gupta Y, Kumar A, Chain PSG, Kovbasnjuk O, Kumar S and Parasher G: MicroRNA in Pancreatic Cancer: From Biology to Therapeutic Potential. Genes (Basel) 10: 752-773, 2019.

12. Zhang B, Guo X, Zhang J, Liu X,Zhan X and LiZ: MicroRNA 224 is downregulated in mucinous cystic neoplasms of the pancreas and may regulate tumorigenesis by targeting Jagged1. Mol Med Rep 10: 3303-3309, 2014.

13. Aaltonen LA and Hamilton SR (eds.): World Health Organization, International Agency for Research on Cancer. Pathology and Genetics of Tumours of the Digestive System. Oxford University Press, Lyon, Oxford, 2000.

14. Li S, Zhang J, Zhao Y, Wang F, Chen Y and Fei X: miR-224 enhances invasion and metastasis by targeting HOXD10 in non-small cell lung cancer cells. Oncol Lett 15: 7069-7075, 2018

15. Zhou J, Hu M, Wang F, Song M, Huang Q and Ge B: miR-224 Controls Human Colorectal Cancer Cell Line HCT116 Proliferation by Targeting Smad4. Int J Med Sci 14: 937-942, 2017.

16. Lu Y, Huang W, Chen H, Wei H, Luo A, Xia G, Deng X and Zhang G: MicroRNA-224, negatively regulated by c-jun, inhibits growth and epithelial-to-mesenchymal transition phenotype via targeting ADAM17 in oral squamous cell carcinoma. J Cell Mol Med 23: 4913-4920, 2019

17. Cullen PK Jr, Remine WH and Dahlin DC: A clinicopathological study of cystadenocarcinoma of the pancreas. Surg Gynecol Obstet 117: 189-195, 1963.

18. Chang R, Song L, Xu Y, Wu Y, Dai C, Wang X, Sun X, Hou Y,Li W, Zhan X, et al: Loss of Wwox drives metastasis in triple-negative breast cancer by JAK2/STAT3 axis. Nat Commun 9: 3486, 2018

19. Song L, Guo J, Chang R, Peng X, Li J, Xu X, Zhan X and Zhan L: LKB1 obliterates Snail stability and inhibits pancreatic cancer metastasis in response to metformin treatment. Cancer Sci 109: 1382-1392, 2018

20. Zhou Y, Chang R, Ji W, Wang N, Qi M, Xu Y, Guo J and Zhan L: Loss of Scribble Promotes Snail Translation through Translocation of HuR and Enhances Cancer Drug Resistance. J Biol Chem 291: 291-302, 2016.

21. Livak KJ and Schmittgen TD: Analysis of relative gene expression data using real-time quantitative PCR and the 2(-Delta Delta C(T)) Method. Methods 25: 402-408, 2001.

22. Hieronymus H, Iaquinta PJ, Wongvipat J, Gopalan A, Murali R, Mao N, Carver BS and Sawyers CL: Deletion of 3p13-14 locus spanning FOXP1 to SHQ1 cooperates with PTEN loss in prostate oncogenesis. Nat Commun 8: 1081-1090, 2017.
23. Hanahan D and Weinberg RA: Hallmarks of cancer: The next generation. Cell 144: 646-674, 2011

24. Huang C, Wang Z, Zhang K, Dong Y, Zhang A, Lu C and Liu L: MicroRNA-107 inhibits proliferation and invasion of laryngeal squamous cell carcinoma cells by targeting CACNA2D1 in vitro. Anticancer Drugs 31: 260-271, 2020.

25. Ma X, Feng J, Lu M, Tang W, Han J, Luo X, Zhao Q, Yang L and Yang L: microRNA-501-5p promotes cell proliferation and migration in gastric cancer by downregulating LPAR1. J Cell Biochem 121: 1911-1922, 2020

26. Ye J, Xie W,Zuo Y, Jing G and Tong J: MicroRNA-496 suppresses tumor cell proliferation by targeting BDNF in osteosarcoma. Exp Ther Med 19: 1425-1431, 2020.

27. Guo C, Peng X, Song L, Ying M, Wu Y, Chang R, Li J, Feng D, Zhan $\mathrm{L}$ and Zhan $\mathrm{X}$ : Autophagy promotes malignant migration and invasion via miR-224-5p/BCL2 in pancreatic mucinous cystadenocarcinoma MCC1 cells. Oncol Lett 20: 276, 2020.

28. Liu H, Li P, Li B, Sun P, Zhang J, Wang B and Jia B: RKIP suppresses gastric cancer cell proliferation and invasion and enhances apoptosis regulated by microRNA-224. Tumour Biol 35: 10095-10103, 2014.

29. Li Q, Ding C, Chen C, Zhang Z, Xiao H, Xie F, Lei L, Chen Y, Mao B, Jiang M, et al: miR-224 promotion of cell migration and invasion by targeting Homeobox D 10 gene in human hepatocellular carcinoma. J Gastroenterol Hepatol 29: 835-842, 2014.

30. Lin ZY, Huang YQ, Zhang YQ, Han ZD, He HC, Ling XH, Fu X, Dai QS, Cai C, Chen JH, et al: MicroRNA-224 inhibits progression of human prostate cancer by downregulating TRIB1. Int J Cancer 135: 541-550, 2014.

31. Brown JA and Bourke E: Practical Bioinformatics Analysis of miRNA Data Using Online Tools. Methods Mol Biol 1509: 195-208, 2017.

32. Lewis BP, Shih IH, Jones-Rhoades MW, Bartel DP, Burge CB and Burge CB: Prediction of mammalian microRNA targets. Cell 115: 787-798, 2003

33. 33. Krek A, Grün D, Poy MN, Wolf R, Rosenberg L, Epstein EJ, MacMenamin P, da Piedade I, Gunsalus KC, Stoffel M, et al: Combinatorial microRNA target predictions. Nat Genet 37: 495-500, 2005.

34. Zhu B and Wei Y: Antitumor activity of celastrol by inhibition of proliferation, invasion, and migration in cholangiocarcinoma via PTEN/PI3K/Akt pathway. Cancer Med 9: 783-796, 2020.

35. Ramírez-Moya J,Wert-Lamas Land SantistebanP:MicroRNA-146b promotes PI3K/AKT pathway hyperactivation and thyroid cancer progression by targeting PTEN. Oncogene 37: 3369-3383, 2018

36. Di Cristofano A and Pandolfi PP: The multiple roles of PTEN in tumor suppression. Cell 100: 387-390, 2000.

37. Lin Y, Chen Q, Liu QX, Zhou D, Lu X, Deng XF, Yang H, Zheng $\mathrm{H}$ and Qiu Y: High expression of DJ-1 promotes growth and invasion via the PTEN-AKT pathway and predicts a poor prognosis in colorectal cancer. Cancer Med 7: 809-819, 2018.

38. Zhao YS, Yang WC, Xin HW, Han JX and Ma SG: miR-182-5p knockdown targeting PTEN inhibits cell proliferation and invasion of breast cancer cells. Yonsei Med J 60: 148-157, 2019.

39. Jiang XM, Yu XN, Liu TT, Zhu HR, Shi X, Bilegsaikhan E, Guo HY, Song GQ, Weng SQ, Huang XX, et al: microRNA-19a-3p promotes tumor metastasis and chemoresistance through the PTEN/Akt pathway in hepatocellular carcinoma. Biomed Pharmacother 105: 1147-1154, 2018.

40. Macgregor-Das AM and Iacobuzio-Donahue CA: Molecular pathways in pancreatic carcinogenesis. J Surg Oncol 107: 8-14, 2013.

This work is licensed under a Creative Commons Attribution-NonCommercial-NoDerivatives 4.0 International (CC BY-NC-ND 4.0) License. 